\title{
Gross motor developmental dysfunctional outcomes in infantile and toddler pediatric intensive care unit survivors
}

\author{
Chun-Feng Yang ${ }^{1}$, Yang Xue ${ }^{2}$, Jun-Yan Feng ${ }^{1}$, Fei-Yong Jia ${ }^{2}$, Yu Zhang ${ }^{2}$ and Yu-Mei Li*
}

\begin{abstract}
Background: Increasing studies have focused on motor function/dysfunction in PICU survivors; however, most studies have focused on adults and older children. This study investigated gross motor developmental function outcomes in infantile and toddler pediatric intensive care unit (PICU) survivors and the factors associated with gross motor developmental functions.
\end{abstract}

Methods: This observational study was conducted in the PICU of the First Hospital of Jilin University between January 2019 and March 2019. Thirty-five eligible patients were divided into the dysfunctional $(n=24)$ or nondysfunctional $(n=11)$ group according to the results of the Peabody Developmental Motor Scales, Second Edition (PDMS-2). Baseline gross motor function for all participants before PICU admission was measured via the Age and Stages Questionnaires, Third Edition (ASQ-3). The PDMS-2 was used to evaluate gross motor development function before PICU discharge.

Results: The gross motor developmental dysfunction incidence was $68.6 \%$. Linear correlation analysis showed that the gross motor quotient (GMQ) was positively correlated with the pediatric critical illness score (PCIS, $r=0.621, P<0.001)$, and negatively correlated with length of PICU stay $(r=-0.556, P=0.001)$, days sedated $(r=-0.602, P<0.001)$, days on invasive mechanical ventilation (IMV; $r=-0.686, P<0.001)$, and days on continuous renal replacement therapy (CRRT; $r=$ $-0.538, P=0.001)$. Linear regression analysis showed that IMV days $(\beta=-0.736, P=0.001)$, sepsis $(\beta=-18.111, P=0.003)$ and PCIS $(\beta=0.550, P=0.021)$ were independent risk factors for gross motor developmental dysfunction.

Conclusions: Gross motor developmental dysfunction in infantile and toddler PICU survivors is more common and may be exacerbated by experiences associated with longer IMV days and increasing illness severity combined with sepsis.

Trial registration: The trial 'Early rehabilitation intervention for critically ill children' has been registered at http://www. chictr.org.cn/showproj.aspx?proj=23132. Registration number: ChiCTR1800020196.

Keywords: Gross motor developmental function, Sepsis, Mechanical ventilation, Pediatric intensive care unit, Infant, Toddler

\section{Introduction}

With improved diagnostic and treatment technology in pediatric critical care medicine, mortality rates have decreased significantly among critically ill children. However, treatments such as invasive mechanical ventilation (IMV), drugs and other factors, can cause newly acquired functional disabilities, in addition to saving the

\footnotetext{
* Correspondence: liyumei1989@126.com

${ }^{1}$ Department of Pediatrics Intensive Care Unit, The First Hospital of Jilin

University, Changchun 130021, China

Full list of author information is available at the end of the article
}

lives of critically ill children [1-3]. Increasing studies have focused on motor function/dysfunction in PICU survival $[1,4,5]$; however, most have focused on adults and older children. Because motor function in older children is similar to that in adults, the methods mostly assess muscle strength, mobility, fatigue and the activities of daily living scale (ADLS), and most assessment results are obtained via questionnaires $[4,6-8]$. However, these assessment methods do not apply to infants and toddlers in the PICU. Because children are not "little adults" [9], their gross motor functions are in the developmental

(c) The Author(s). 2019 Open Access This article is distributed under the terms of the Creative Commons Attribution 4.0 International License (http://creativecommons.org/licenses/by/4.0/), which permits unrestricted use, distribution, and 
stage, which is a critical period for gaining motor skills. Therefore, assessing gross motor developmental function can better reflect the physical functions of infants and toddlers.

Few studies have focused on acquired gross motor developmental dysfunction in children. Hövels-Gürich et al. [10] found that the neonatal arterial switch operation with combined circulatory arrest and low-flow bypass was associated with neurological and fine and gross motor impairment. In addition, gross motor developmental function assessment is mostly used in neonatal intensive care units (NICUs) and in high-risk infants [11, 12]. Patients are at different risks for having developmental dysfunction between NICU and PICU, therefore, the two populations differ entirely [13, 14]. Therefore, our study investigated the gross motor developmental function outcomes of infantile and toddler survivors of pediatric intensive care units (PICUs) and is the first to assess gross motor developmental function in infants and toddlers in a PICU.

\section{Materials and methods Patients}

This observational study was conducted in the pediatric intensive care unit of the First Hospital of Jilin University, ChangChun, China. Eligible cases were children aged between 1 month and 3 years who were hospitalized in the PICU $\geq 48$ h between January 2019 and March 2019, and for whom it was their first PICU admission during the study period. Children were excluded if they had neuromuscular junction disease, central nervous system disease, limb fractures or deep vein thrombosis. Children with gross motor developmental dysfunction before PICU admission were also excluded. The hospital's ethics committee granted permission for the study, and the eligible children's parents/guardians provided written informed consent. The trial was registered at clinical trials.gov (ChiCTR1800020196). All participants' information sheets were provided to their parents.

\section{Procedure}

The PICU cohort was categorized into two groups: the dysfunctional group $(\mathrm{GMQ}<90)$ and the nondysfunctional group $(G M Q \geq 90)$. Age, sex, diagnosis, severity of illness, length of PICU stay, days on IMV, days on methylprednisolone, days sedated, days on continuous renal replacement therapy (CRRT), and application of vasoactive drugs were recorded for each group. Because of the sample size, primary diagnoses were broadly categorized as cardiovascular, respiratory, gastrointestinal, or other (genitourinary, hematologic/oncologic, musculoskeletal, endocrinologic, or trauma). Baseline gross motor function was measured for all participants before PICU admission using the Age and Stages Questionnaires, Edition 3 (ASQ-3) to assess the gross motor developmental function of participants prior to PICU admittance. At the time of PICU discharge, all patients completed the Peabody Developmental Motor Scales, Second Edition (PDMS-2), which assesses gross motor development function $[15,16]$. To ensure the accuracy of the assessment results, one experienced physiotherapist assessed all participants in an assessment room that met the conditions for motor assessment.

\section{Measures}

Illness severity was measured using the pediatric critical illness score (PCIS) [17] developed by the Chinese Medical Association Emergency Department and the Chinese Medical Association Emergency Society Pediatrics Group. The PCIS is currently the most widely used pediatric critical illness scoring method in China, and it's total score is negatively correlated with the severity of the disease which can accurately determine the condition and predict the risk of death in children [18].

The ASQ is a reliable, standardized, parent-completed, developmental screening test composed of 21 agespecific questions covering the ages of 1-66 months [19]. And it is a reliable and valid measure which can be used to screen and monitor the development of children in the mainland of China [20]. The ASQ-3 encompasses five developmental areas: communication, gross motor, fine motor, problem solving and personal-social [21, 22]. Three responses are possible per item, depending on whether the child can perform the task: "Yes" (10points), "Sometimes" (5 points) and "Not Yet" (0 points). The total score for each area is obtained by adding the scores of the six items. The assessment results are divided into normal, critical and abnormal based on each area's total score. Only those children with normal results of ASQ were enrolled in our study.

At the time of PICU discharge, the patients' gross motor developmental function was measured using the PDMS-2, which is a norm-referenced tool designed to assess the fine and gross motor skills of children aged between 0 and 71 months. Its normative sample was based on 2003 children in 46 states of the United States and one Canadian province [23]. The PDMS-2 is suitable for assessing various populations of children at high risk for motor delays. It has a high degree of reliability and validity regarding child development in China [24-26], but few studies have applied the PDMS-2 to children in PICUs. The PDMS-2 is composed of four subtests: reflex, stationary, locomotion, and object manipulation, and each subtest contributes to the gross motor quotient (GMQ) score. The scores are interpreted as very superior (131-165), superior (121$130)$, above average (110-120), average (90-109), below average (80-89), poor (70-79), or very poor (35-69) [27]. Gross motor developmental dysfunction was defined as GMQ $<90$ [27]. 


\section{Statistical analyses}

Data were analyzed using IBM SPSS Statistics for Windows, version 22.0 (IBM Corp, Armonk, NY, USA). Continuous variables are described as the mean $\pm \mathrm{SD}$ or median (interquartile range), depending on whether the distribution was normal or non-normal. Normality test of variables using Shapiro-Wilk test. Categorical variables are described as $n$ (\%). Continuous variables were compared using Student's ttest or the Mann-Whitney $U$ test. Categorical variables were compared using the chi-square test or Fisher's exact test depending on sample size. For the correlation analyses, the Spearman method was used to test the relationship between GMQ and PCIS, length of PICU stay, IMV days, days on methylprednisolone, days sedated and CRRT days. The relationship among multiple factors was analyzed via multivariate linear regression (stepwise method), and the dependent variable, $\mathrm{Y}$, was a continuous variable with a normal distribution. For all final comparisons, $P \leq 0.05$ was considered statistically significant.

\section{Results}

\section{Sample characteristics}

During the study period, 70 of 105 PICU patients were excluded. Of these 70 patients, 8 had motor developmental delays before entering the PICU, 18 had parents who refused to give permission for the motor development assessment, 10 died during PICU hospitalization, 19 had abnormal ASQ-3 results, and 15 were discharged within $48 \mathrm{~h}$ of PICU admittance. Finally, 35 patients met the inclusion criteria. Participants were categorized as either children with developmental dysfunction $(n=24)$ or children without developmental dysfunction $(n=11)$ based on whether the GMQ was $\geq$ or $<90$ according to the results of PDMS-2 (Fig. 1).

Age, sex, diagnostic category, percentage of surgical patients, percentage of sepsis, use of vasoactive medications, PCIS, length of PICU stay, IMV days, days on sedatives, days on methylprednisolone and CRRT days were recorded for both groups (Table 1). Of all 35 eligible patients, 24 had gross motor developmental dysfunction with an incidence of $68.6 \%$ (24/35). PCIS in the dysfunctional group was significantly lower than that in the nondysfunctional group $(81.0 \pm 89.09$ vs $92.18 \pm 5.17, P=$ $0.001)$. Use of vasoactive medications $(58.2 \%$ vs $8.2 \%, P=$ 0.006), length of PICU stay (22.50 [range, 14.50-27.75] vs 9.00 [7.00-17.00], $P=0.029)$, days on sedatives (9.0 [7.0$13.0]$ vs $1.0[0.3-2.0], P=0.021)$, and IMV days $(7.46 \pm$ 5.34 vs $1.27 \pm 2.83, P=0.001)$ were significantly higher than those parameters in the non- dysfunctional group.

Linear correlation between GMQ and PCIS, length of PICU stay, IMV days, days on sedatives, days on methylprednisolone, and CRRT days

We performed a linear correlation analysis between the GMQ and PCIS, length of stay in the PICU, IMV days,

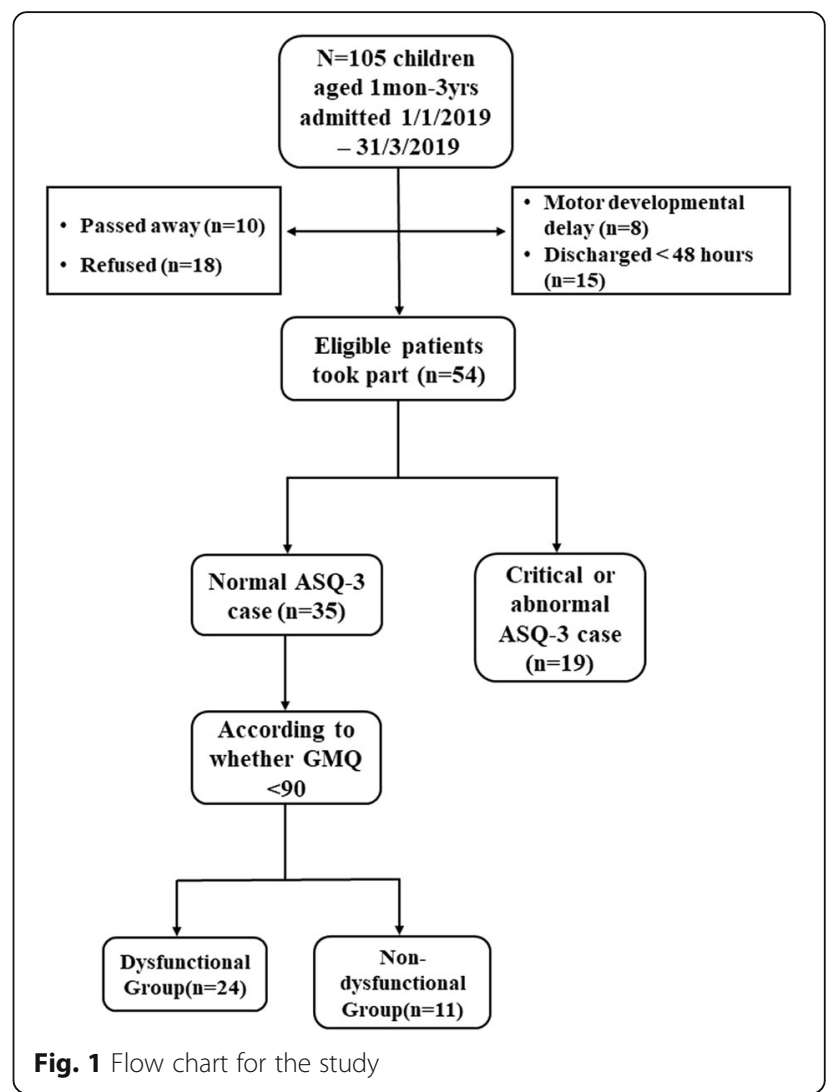

days on sedatives, days on methylprednisolone, and CRRT days. The results showed that GMQ was positively correlated with PCIS $(r=0.621, P<0.001)$, while length of PICU stay $(r=-0.556, P=0.001)$, days sedated $(r=-0.602, P<0.001)$, IMV days $(r=-0.686, P<0.001)$, and CRRT days $(r=-0.538, P=0.001)$ were negatively correlated with the GMQ (Fig. 2a-f).

Linear regression analysis between GMQ and length of PICU stay, days on sedatives, IMV days, CRRT days, PCIS, use of vasoactive medications, and sepsis

We performed a linear regression analysis of the above factors and the percentages of sepsis and vasoactive drugs with the GMQ. The results showed that sepsis $(\beta=-18.11, P=0.003)$, PCIS $(\beta=0.55, P=0.021)$ and IMV days $(\beta=-0.736, P=0.001)$ were independent risk factors for a decreased GMQ (Table 2).

\section{Discussion}

The infant and toddler periods are vital times for gross motor development in humans. Gross motor behavior is one of the earliest directly observable elements of adaptive function. At 12 months old, children begin learning to walk, and their functional motor connectivity is correlated with walking [28]. During the toddler years, children change quickly in their motor function and physical growth, and their motor skills and ability to 
Table 1 Participants Study Cohort Characteristics

\begin{tabular}{llll}
\hline Characteristics & $\begin{array}{l}\text { Dysfunction } \\
\text { Group } \\
(n=24)\end{array}$ & $\begin{array}{l}\text { Non- Dysfunction } \\
\text { Group } \\
(n=11)\end{array}$ \\
\hline Age, months (mean \pm SD) & $20.9 \pm 12.4$ & $15.6 \pm 12.9$ & $63.6(7 / 11)$ \\
Male sex (\%) & $58.3(14 / 24)$ & & \\
Reason for admission (\%) & & $9.1(1 / 11)$ & 0.005 \\
$\quad$ Cardiovascular & $8.3(2 / 24)$ & $81.8(9 / 11)$ & 0.766 \\
Respiratory & $83.3(20 / 24)$ & $9.1(1 / 11)$ & 0.941 \\
Gastrointestinal & $8.3(2 / 24)$ & $92.18 \pm 5.17$ \\
PCIS (mean \pm SD) & $81.08 \pm 9.09$ & $9.00(7.00-17.00)$ \\
PICU length of stay, d, median (IQR) & $22.50(14.50-27.75)$ & $8.2(9 / 11)$ & 0.912 \\
Use of vasoactive medications, n (\%) & $58.2(14 / 24)$ & $1.27 \pm 2.83$ \\
IMV days (mean \pm SD) & $7.46 \pm 5.34$ & $2.0(1.0-7.0)$ & 0.941 \\
Sedative days, d, median (IQR) & $8.50(2.50-13.75)$ & $3.00(0.00-7.00)$ \\
Methylprednisolone, d, median (IQR) & $5.5(4.25-7.75)$ & & 0.001 \\
CRRT days, d, median (IQR) & $3.50(0.00-7.00)$ & $9.1(1 / 11)$ & 0.006 \\
Sepsis (\%) & $20.8(5 / 24)$ & & 0.001 \\
Surgical, $n$ (\%) & $8.3(2 / 24)$ & 0.130 \\
\hline
\end{tabular}

$I Q R$ interquartile range, $S D$ standard deviation, $P C I S$ pediatric critical illness score, $C R R T$ continuous renal replacement therapy, IMV invasive mechanical ventilation, $G M Q$ gross motor quotient

explore their environment improve [29]. Therefore, at this stage, factors such as diseases, environment and nutrition, interfere with the chances of infants and young children receiving external information, which affects their motor development. Uzark et al. [30] found that gross motor impairments were common in infants after cardiac operations. Friedman et al. [31] showed that young (aged 1-3 years) congenital diaphragmatic hernia survivors continued to have a high incidence of motor and language problems. Although motor developmental dysfunction presents in these populations, the motor developmental function levels of children in the PICU remain unclear. In addition, children in PICUs are more critically ill, undergo more invasive procedures, and receive more drugs; therefore, the level of motor developmental function in these infants and toddlers deserves attention. Unfortunately, few studies have focused on infants and toddlers in PICUs. This study was the first to assess gross motor developmental function in PICU survivors.

In this study, the incidence of gross motor developmental dysfunction was $68.6 \%$. This statistic is higher than that reported for children after cardiac surgery (incidence $21-64 \%$ ) [30, 32]. The incidence of motor dysfunction was $60 \%$ in infants who survived congenital diaphragmatic hernia repair [31], possibly because of the longer mechanical ventilation time, ICU stay, and more complications in infants and toddlers in the PICU compared with those with post-cardiac surgery. We believe that for critically ill children aged 1 month to 3 years, assessing gross motor developmental function is more important in guiding subsequent rehabilitation.

We also found that IMV days were significantly longer in the dysfunctional group than in the non- dysfunctional group. This suggests that the length of IMV is related to the occurrence of gross motor developmental dysfunction. IMV is one of the most commonly used treatment methods in PICUs, but it causes many dysfunctions in motor skills, cognition and psychology despite saving the lives of critically ill children. At present, many studies in adults have shown that IMV is a high-risk factor for ICUacquired weakness (AW) [33, 34]. Patel et al. [35] found that patients with ICU-AW had significantly longer mechanical ventilation times. A systematic review of published work showed evidence of ICU-AW in 46\% (95\% confidence interval [CI] 43-49\%) of adult ICU patients who experienced lengthy mechanical ventilation, sepsis, or multiorgan failure [36]. However, the effects of mechanical ventilation on infants' gross motor developmental levels remain unreported. Our research showed that IMV days were associated with infants' gross motor developmental dysfunction. IMV is also accompanied by longer PICU stays, more sedative use, more severe protopathic conditions and more invasive examinations. Length of PICU stay, days on sedatives, and days using CRRT were longer, and the vasoactive drug use rate, sepsis incidence and PCIS scores were higher in the dysfunctional group than in the nondysfunctional group. These factors may promote gross motor developmental dysfunction in children. 

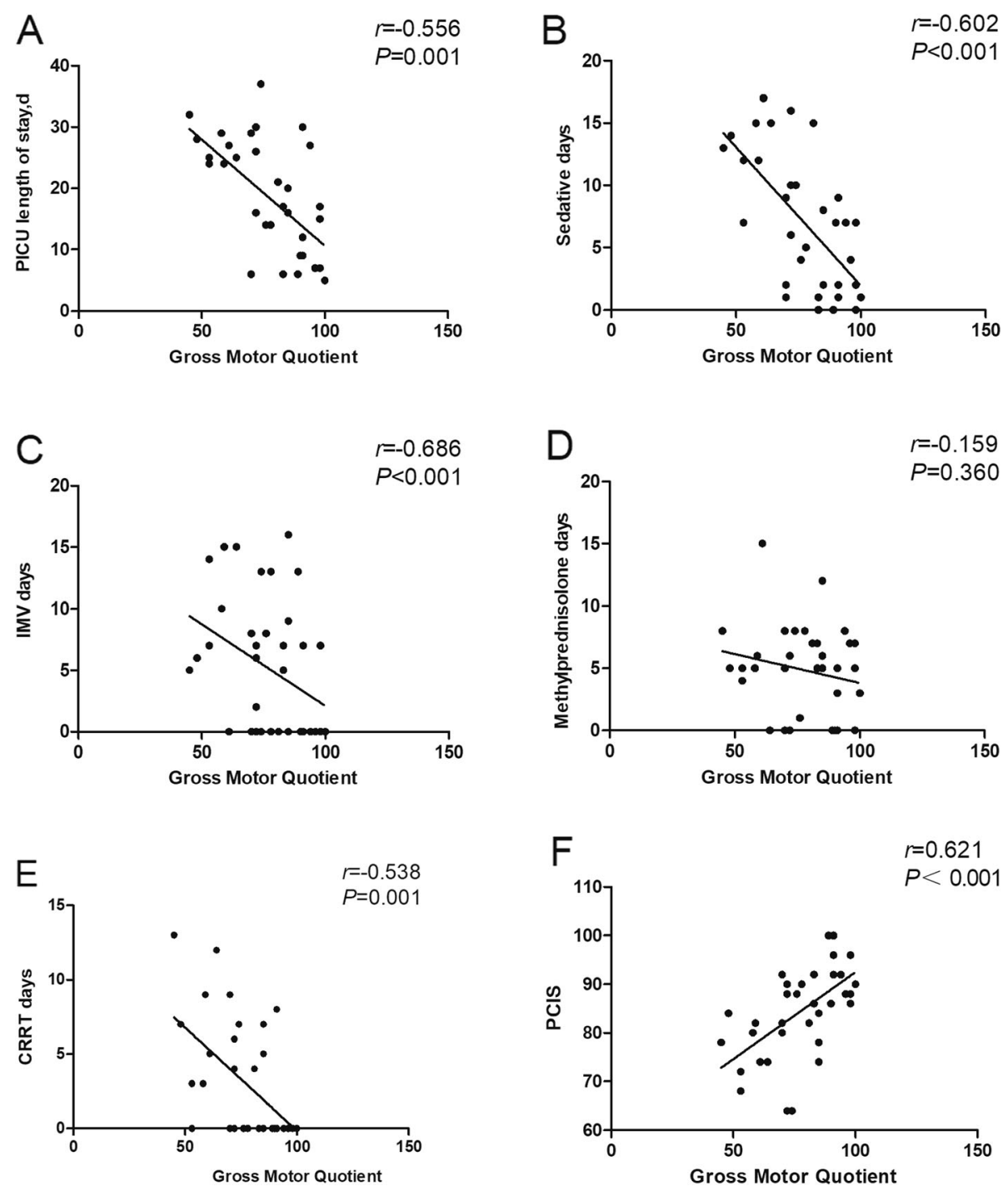

Fig. 2 a-f Spearman's correlation test was used to evaluate the relationships between GMQ and length of PICU stay, days on sedatives, IMV days, days on methylprednisolone, CRRT days and PCIS, respectively

Our research showed that the above factors were linearly correlated with GMQ, and the degree of gross motor dysfunction was significantly negatively correlated with PICU stay, sedative use and CRRT days and positively associated with PCIS scores. The GMQ of septic

Table 2 Linear regression analysis of risk factors associated with GMQ

\begin{tabular}{llll}
\hline Factors & \multicolumn{3}{l}{ Gross Motor Quotient } \\
\cline { 2 - 4 } & $\beta$ & $\operatorname{Se}(\beta)$ & $P$ \\
\hline IMV days & -0.736 & 0.260 & 0.001 \\
Sepsis & -18.110 & 0.414 & 0.003 \\
PCIS & 0.550 & 0.338 & 0.021 \\
\hline
\end{tabular}

The risk factors included in the linear regression analysis were length of PICU stay, days on sedatives, IMV days, CRRT days, PCIS, use of vasoactive medications and sepsis patients was also significantly lower than that of aseptic patients. To further analyze the independent risk factors that lead to gross motor developmental dysfunction, we performed a linear regression analysis of the above factors. The results showed that IMV days, sepsis and PCIS are independent risk factors for gross motor developmental dysfunction in PICU infants and toddlers. This is consistent with the results of an adult study on ICU-AW. Jongheet al [37]. conducted a multicenter, prospective study that showed that physical dysfunction in ICU patients was associated with prolonged mechanical ventilation. A prospective cohort study by Borges et al. indicated that physical activity, exercise capacity, and muscle strength were significantly reduced in ICU sepsis survivors, even at 3 months after discharge [38]. A meta-analysis conducted by Yang et al. incorporating 14 studies, showed that sepsis 
(OR, 2.2; 95\%CI, 1.30-3.71) and duration of IMV (OR, 1.1; 95\%CI, 1.00-1.22) were significantly associated with ICU-AW [39]. A multicenter study by Choong et al. suggested that Pediatric Risk of Mortality III (PRISM III) is an independent risk factor of social/cognitive dysfunction [40].

To investigate the independent risk factors for gross motor dysfunction in infants and Toddlers, we used a multivariate linear regression analysis. The result showed that IMV days, sepsis and PCIS were independent risk factors for gross motor developmental dysfunction. This is similar to the results of several adult studies $[41,42]$. We believe that the above risk factors leading to children's gross motor developmental dysfunction may have three pathways: 1 . Prolonged mechanical ventilation and sepsis can lead to limb muscle atrophy [43, 44], resulting in weakened muscle strength in children; thus, abnormal assessment results may be due to weakened muscle strength; 2. Studies have confirmed that sepsis and IMV can cause brain dysfunction $[45,46]$. However, the central nervous systems of infants and young children remain in the developmental stage, and motor neuron integrity is crucial to children's mastering their motor skills. Therefore, damage to the child's motor center due to sepsis and IMV days may affect the overall motor developmental level. 3. Impairment of cognitive function is related to motor developmental dysfunction, and previous studies have confirmed the effects of sepsis and mechanical ventilation on cognitive function $[43,47,48]$. Impaired cognitive function factors can affect motor function in children, especially infants $[49,50]$. Whether this phenomenon exists in infantile and toddler PICU survivors requires further study.

This study had several limitations. First, the study was an observational study with a small sample size. Therefore, we found that only IMV days, sepsis and PCIS differed statistically when performing linear regression analyses on related factors. Previous studies showed that hormone and sedative use were significantly associated with the occurrence of physical dysfunction in pediatric and adult patients [51-53]. This study yielded inconsistent results; Therefore, more samples should be included for further analysis. Second, two assessment methods were used to assess the same patient pre-PICU and post-PICU. Due to the children's pre-admission GMQs were unavailable, only the ASQ-3 questionnaire could be used to indirectly reflect gross motor function. Third, our study only assessed the participants' motor development, while infant and toddler PICU survivors may have other developmental impairments such as cognitive, speech, psychological, and emotional disorders. Whether these dysfunctions are related to motor dysfunction warrants further study. Finally, we did not follow-up the enrollees to observe their gross motor function after discharge. In the next study, we will further follow this.
In conclusion, this study showed that gross motor developmental dysfunction in infantile and toddler PICU survivors are more common and may be exacerbated by experiences associated with longer IMV days and increasing illness severity combined with sepsis.

We suggest that early rehabilitative intervention in these children's gross motor developmental function may reduce physical morbidity. Furthermore, detailed comprehensive investigations of developmental functions, including gross motor, fine motor, language, cognition and social abilities, are warranted.

\section{Abbreviations \\ ADLS: Activities of Daily Living Scale; ASQ-3: Age and Stages Questionnaires, Edition 3; CRRT: Continuous renal replacement therapy; GMQ: Gross motor quotient; ICU-AW: Intensive care unit-acquired weakness; IMV: Invasive mechanical ventilation; MRC: MedicalResearch Council; NICU: Neonatal intensive care unit; PCIS: Pediatric critical illness score; PDMS-2: Peabody Developmental Motor Scales, Second Edition; PICU: Pediatric intensive care unit; PRISM III: Pediatric Risk of Mortality III}

\section{Acknowledgements}

The authors thank the study site staff for their cooperation. The participation of the parents and children in this study is greatly acknowledged.

\section{Authors' contributions}

CFY conceived the study design and participated in study management. YX participated in the study design. JYF and YZ performed statistical analyses. FYJ and YM L conceived and designed the study. All authors interpreted the data, contributed to the intellectual content, reviewed the manuscript, and approved the final version.

\section{Funding}

This work received no financial support.

\section{Availability of data and materials}

The datasets used and/or analyzed during the current study are available from the corresponding author on reasonable request.

\section{Ethics approval and consent to participate}

The institutional ethics committee of the First Hospital of Jilin University (ChiCTR1800020196) approved the study. The parents or guardians of the eligible children provided written informed consent. An information sheet was provided for the parents or guardians of all participants.

\section{Consent for publication}

Not applicable.

\section{Competing interests}

The authors declare that they have no competing interests.

\section{Author details}

'Department of Pediatrics Intensive Care Unit, The First Hospital of Jilin University, Changchun 130021, China. ${ }^{2}$ Department of Developmental and Behavioral Pediatrics, The First Hospital of Jilin University, Changchun, China.

Received: 19 September 2019 Accepted: 16 December 2019

Published online: 21 December 2019

\section{References}

1. Field-Ridley A, Dharmar M, Steinhorn D, et al. ICU-acquired weakness is associated with differences in clinical outcomes in critically ill children. Pediatr Crit Care Med. 2016;17(1):53-7.

2. Esses SA, Small S, Rodemann A, et al. Post-intensive care syndrome: educational interventions for parents of hospitalized children. Am J Crit Care. 2019;28(1):19-27.

3. Newth $\mathrm{CIL}$, Khemani RG, Jouvet PA, et al. Mechanical ventilation and decision support in pediatric intensive care. Pediatr Clin N Am. 2017;64(5):1057-70. 
4. Als LC, Picouto MD, Hau SM, et al. Mental and physical well-being following admission to pediatric intensive care. Pediatr Crit Care Med. 2015;16(5):e141-9.

5. Pinto NP, Rhinesmith EW, Kim TY, et al. Long-term function after pediatric critical illness: results from the survivor outcomes study. Pediatr Crit Care Med. 2017;18(3):e122-30

6. Colville GA, Pierce CM. Children's self-reported quality of life after intensive care treatment. Pediatr Crit Care Med. 2013;14(2):e85-92.

7. Herrup EA, Wieczorek B, Kudchadkar SR. Characteristics of postintensive care syndrome in survivors of pediatric critical illness: a systematic review. World J Crit Care Med. 2017:6(2):124-34.

8. Parry SM, Huang M, Needham DM. Evaluating physical functioning in critical care: considerations for clinical practice and research. Crit Care. 2017;21(1):249.

9. Schindler MB. Prediction of ventilation weaning outcome: children are not little adults. Crit Care. 2005;9(6):651-2

10. Hovels-Gurich HH, Seghaye MC, Dabritz S, et al. Cognitive and motor development in preschool and school-aged children after neonatal arterial switch operation. J Thorac Cardiovasc Surg. 1997;114(4):578-85.

11. Subedi D, DeBoer MD, Scharf RJ. Developmental trajectories in children with prolonged NICU stays. Arch Dis Child. 2017;102(1):29-34.

12. Ricci $E_{1}$ Einspieler $C_{1}$ Craig AK. Feasibility of using the general movements assessment of infants in the United States. Phys Occup Ther Pediatr. 2018; 38(3):269-79.

13. Byrne $\mathrm{E}$, Garber J. Physical therapy intervention in the neonatal intensive care unit. Phys Occup Ther Pediatr. 2013;33(1):75-110.

14. Pollack MM, Holubkov R, Funai T, et al. Pediatric intensive care outcomes: development of new morbidities during pediatric critical care. Pediatr Crit Care Med. 2014;15(9):821-7.

15. Fay D, Wilkinson T, Anderson AD, et al. Effects of Modified Instructions on Peabody Developmental Motor Scales, Second Edition, Gross Motor Scores in Children with Typical Development. Phys Occup Ther Pediatr. 2019;39(4):433-45

16. Tso WWY, Wong VCN, Xia $X$, et al. The Griffiths development scales-Chinese (GDS-C): a cross-cultural comparison of developmental trajectories between Chinese and British children. Child Care Health Dev. 2018;44(3):378-83.

17. Zhang $L$, Huang $H$, Cheng $Y$, et al. Predictive value of four pediatric scores of critical illness and mortality on evaluating mortality risk in pediatric critical patients. Zhonghua Wei Zhong Bing Ji Jiu Yi Xue. 2018;30(1):51-6.

18. Fan XM, PT Collaborative Group. For simplified. Clinical application of simplified pediatric critical illness scoring system. Zhong Hua Er Ke Za Zhi. 2003;41(8):565-9.

19. Halbwachs M, Muller JB, Tich SNT, et al. Usefulness of parent-completed ASQ for neurodevelopmental screening of preterm children at five years of age. PLoS One. 2013;8(8):e71925.

20. Wei M, Bian X, Squires J, et al. Studies of the norm and psychometrical properties of the ages and stages questionnaires, third edition, with a Chinese national sample. Zhong Hua Er Ke Za Zhi. 2015;53(12):913.

21. Steenis $\sqcup$, Verhoeven $M$, Hessen DJ, et al. Parental and professional assessment of early child development: the ASQ-3 and the Bayley-III-NL. Early Hum Dev. 2015;91(3):217-25.

22. Marks KP, Madsen Sjö N, Wilson P. Comparative use of the Ages and Stages Questionnaires in the USA and Scandinavia: a systematic review. Dev Med Child Neurol. 2019;61(4):419-30.

23. Saraiva $L$, Rodrigues LP, Cordovil $R$, et al. Motor profile of Portuguese preschool children on the Peabody developmental motor Scales-2: a crosscultural study. Res Dev Disabil. 2013;34(6):1966-73.

24. Tavasoli A, Azimi P, Montazari A. Reliability and validity of the Peabody developmental motor scales-second edition for assessing motor development of low birth weight preterm infants. Pediatr Neurol. 2014;51(4):522-6.

25. Glass TJA, Chau V, Grunau RE, et al. Multiple postnatal infections in newborns born preterm predict delayed maturation of motor pathways at term-equivalent age with poorer motor outcomes at 3 years. J Pediatr. 2018;196:91-97 e1.

26. Wang HH, Liao HF, Hsieh CL. Reliability, sensitivity to change, and responsiveness of the Peabody developmental motor scales-second edition for children with cerebral palsy. Phys Ther. 2006;86(10):1351-9.

27. Maddox T. Peabody developmental motor scales. In: Encyclopedia of Special Education. 2nd ed; 2008.

28. Marrus N, Eggebrecht AT, Todorov A, Elison JT, Wolff JJ, Cole L, et al. Walking, gross motor development, and brain functional connectivity in infants and toddlers. Cereb Cortex. 2018;28(2):750-63.

29. Colson ER, Dworkin PH. Toddler development. Pediatr Rev. 1997;18(8):255-9.
30. Uzark K, Smith C, Donohue J, Yu S, Romano JC. Infant motor skills after a cardiac operation: the need for developmental monitoring and care. Ann Thorac Surg. 2017; S0003497516318902.

31. Friedman S, Chen C, Chapman JS, Jeruss S, Terrin N, Tighiouart H, et al. Neurodevelopmental outcomes of congenital diaphragmatic hernia survivors followed in a multidisciplinary clinic at ages 1 and 3. J Pediatr Surg. 2008;43(6):0-1043.

32. Hamrick SEG, Gremmels DB, Keet CA, Leonard CH, Connell JK, Hawgood S, et al. Neurodevelopmental outcome of infants supported with extracorporeal membrane oxygenation after cardiac surgery. Pediatrics. 2003:111(6 Pt 1):e671-5.

33. Friedrich $\mathrm{O}$, Reid MB, Van den Berghe $\mathrm{G}$, et al. The sick and the weak: neuropathies/myopathies in the critically ill. Physiol Rev. 2015;95(3):1025-109.

34. Larsson L, Friedrich O. Critical illness myopathy (CIM) and ventilator-induced diaphragm muscle dysfunction (VIDD): acquired myopathies affecting contractile proteins. Compr Physiol. 2016;7(1):105-12.

35. Patel BK, Pohlman AS, Hall JB, et al. Impact of early mobilization on glycemic control and ICU-acquired weakness in critically ill patients who are mechanically ventilated. Chest. 2014;146(3):583-9.

36. Latronico N, Bolton CF. Critical illness polyneuropathy and myopathy: a major cause of muscle weakness and paralysis. Lancet Neurol. 2011;10(10):931-41.

37. De Jonghe $B$, Sharshar $T$, Lefaucheur JP, et al. Paresis acquired in the intensive care unit: a prospective multicenter study. JAMA. 2002;288(22): 2859-67.

38. Borges RC, Carvalho CR, Colombo AS, et al. Physical activity, muscle strength, and exercise capacity 3 months after severe sepsis and septic shock. Intensive Care Med. 2015;41(8):1433-44.

39. Yang $T$, Li Z, Jiang $L$, et al. Risk factors for intensive care unit-acquired weakness: a systematic review and meta-analysis. Acta Neurol Scand. 2018; 138(2):104-14.

40. Choong K, Fraser D, Al-Harbi S, et al. Functional recovery in critically ill children, the "WeeCover" multicenter study. Pediatr Crit Care Med. 2018; 19(2):145-54.

41. Mehta AB, Walkey AJ, Curran-Everett D, et al. Hospital mechanical ventilation volume and patient outcomes: too much of a good thing? Crit Care Med. 2019;47(3):360-8.

42. Arah OA. Hospital volume and outcomes of mechanical ventilation. N Engl J Med. 2006;355(15):1617 author reply 1617-1619.

43. Ji M, Yuan H, Yuan S, et al. The p75 neurotrophin receptor might mediate sepsis-induced synaptic and cognitive impairments. Behav Brain Res. 2018; 347:339-49.

44. Baldwin CE, Bersten AD. Myopathic characteristics in septic mechanically ventilated patients. CurrOpin Clin Nutr Metab Care. 2015;18(3):240-7.

45. Adam N, Kandelman S, Mantz J, et al. Sepsis-induced brain dysfunction. Expert Rev Anti-Infect Ther. 2013;11(2):211-21.

46. Sonneville $R$, Verdonk $F$, Rauturier $C$, et al. Understanding brain dysfunction in sepsis. Ann Intensive Care. 2013;3(1):15.

47. Girard TD, Thompson JL, Pandharipande PP, et al. Clinical phenotypes of delirium during critical illness and severity of subsequent long-term cognitive impairment: a prospective cohort study. Lancet Respir Med. 2018 . 6(3):213-22.

48. Patel MB, Morandi A, Pandharipande PP. What's new in post-ICU cognitive impairment? Intensive Care Med. 2015;41(4):708-11.

49. Champion JA, Rose KJ, Payne JM, et al. Relationship between cognitive dysfunction, gait, and motor impairment in children and adolescents with neurofibromatosis type 1. Dev Med Child Neurol. 2014;56(5):468-74.

50. Al-Nemr A, Abdelazeim F. Relationship of cognitive functions and gross motor abilities in children with spastic diplegic cerebral palsy. Appl Neuropsychol Child. 2018;7(3):268-76.

51. Meduri GU, Schwingshackl A, Hermans G. Prolonged Glucocorticoid Treatment in ARDS: Impact on Intensive Care Unit-Acquired Weakness. Front Pediatr. 2016:4:69.

52. Taipale HT, Bell JS, Gnjidic D, et al. Muscle strength and sedative load in community-dwelling people aged 75 years and older: a population-based study. J Gerontol A Biol Sci Med Sci. 2011;66(12):1384-92.

53. Tomita S, Matsuura N, Ichinohe T. The combined effects of midazolam and propofol sedation on muscle power. Anaesthesia. 2013;68(5):478-83.

\section{Publisher's Note}

Springer Nature remains neutral with regard to jurisdictional claims in published maps and institutional affiliations. 